\title{
NUMERICAL PREDICTIONS AND MECHANICAL TESTING OF BRAIDED COMPOSITE STRUCTURES UTILISING DIGITAL IMAGE CORRELATION
}

\author{
Emil Pitz*, Matei-Constantin Miron, Imre Kállai, Zoltán Major \\ Institute of Polymer Product Engineering, Johannes Kepler Universität Linz, Altenberger Straße 69, Linz, \\ Austria, 4040 \\ * corresponding author: emil.pitz@jku.at
}

Abstract. The current paper is describing the implementation of a multiscale numerical model for prediction of stiffness and strength in braided composites. The model is validated by experimental testing of single-layer braided tubes under torsional loading utilising digital image correlation (DIC).

For the numerical model the entire braided structure is modelled at yarn detail level, taking into account the yarn behaviour as well as individual yarn-to-yarn interactions by using cohesive contact definitions. By means of Hashin's failure criteria and cohesive contact damage, failure of the yarns and failure of the yarn-to-yarn interface is being accounted for. Thereby the material failure behaviour can be predicted.

For validation of the model, torsion tests of biaxially braided single-layer composite tubes were performed. The strain distribution at the specimen surface was studied using the DIC system ARAMIS in $3 \mathrm{D}$ mode.

KEYWORDS: braided composites, finite element analysis, failure prediction, torsion testing, digital image correlation.

\section{INTRODUCTION}

Aim of this research is the implementation and validation of a numerical tool providing reliable predictions of the mechanical properties of braided composite materials. Modelling the structure at yarn detail level (meso-level), the undulation of the yarns is taken into account as well as individual yarn-to-yarn interactions, utilising cohesive contact interactions in the finite element software Abaqus. By implementation of failure models, the approach can predict occurring failure mechanisms, distinguishing damage at the yarn level (intra-yarn) and yarn-interface level (inter-yarn). Material properties required as input parameters are obtained by mean-field homogenization ( $\mathrm{MFH}$ ) from the individual constituents behaviour (matrix and fibres).

For validation of the model, single layer braided tubes are tested under torsional loading utilising DIC for full-field strain measurement on the surface of the specimen.

The global loading response predicted by the numerical model as well as the local strain distribution and occurring instability behaviour is compared to the experimentally obtained results.

\section{Numerical Model}

Generally three structural levels can be distinguished in textile composites: The macro-level, defining the overall 3D geometry of the composite structure, the meso-level, defining the internal structure of the reinforcement, consisting of the impregnated undulating yarns and resin pockets corresponding to the empty space between the yarns, and the micro-level, defining the arrangement of the fibre filaments in the impregnated yarn [1]. A schematic representation of the multiscale problem can be seen in Figure 1 .

To describe this structure in the numerical model, a multiscale modelling approach was utilised, describing the entire structure at yarn-detail level and distinguishing between intra-yarn (fibre-dominated) and inter-yarn (matrix-dominated) behaviour [3, 4].

\subsection{D-Model of the Braided Composite} In order to achieve the mentioned degree of accuracy, a 3D-model of the braiding geometry was developed, modelling both the yarn undulation path and the yarn cross section.

The path of each undulating yarn was analytically approximated by a tapered cosine function:

$$
r(x)=r_{0}-\frac{t}{2} \sin \left(\frac{\pi}{2} \cos (x-\delta)\right) .
$$

The phase shift $\delta$ is changing periodically for each yarn path, depending on the modelled weave pattern (plan or twill), leading to interwoven and repeating undulation paths (see Figure 2).

The yarn cross section shape is linked to the shape of the yarn path. In this approach, it is taken from projection of the undulation path onto a plane orthogonal to the braiding direction.

For simulation, mid-plane shell bodies are extracted from the solid geometry and the local thickness is mapped on the shell mesh. 


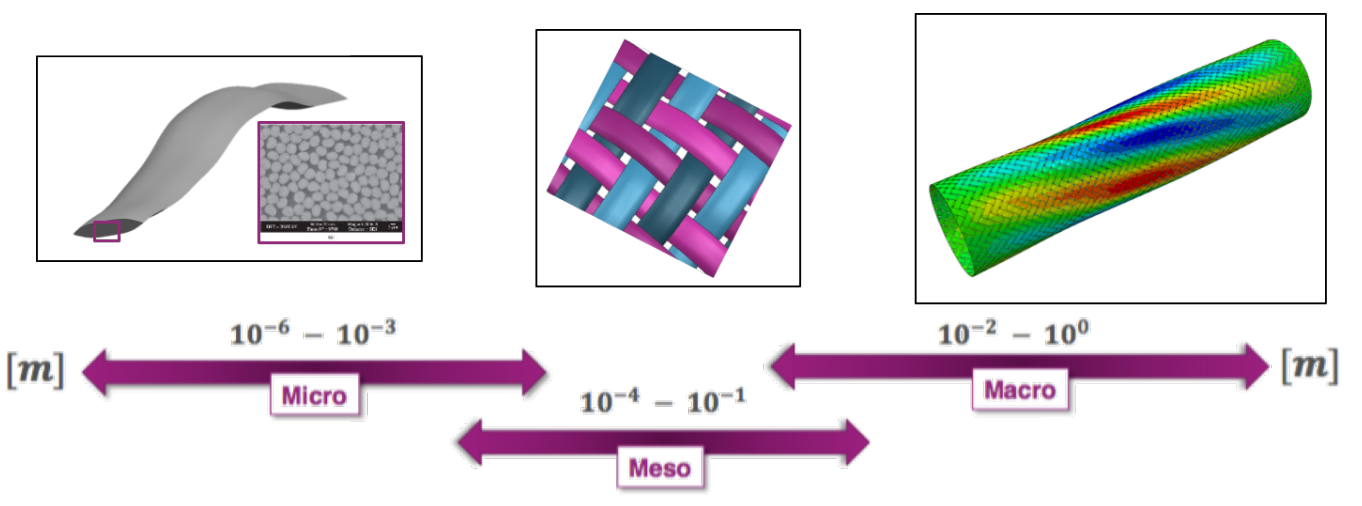

Figure 1. Structural levels of a textile composite. (adapted from [2])

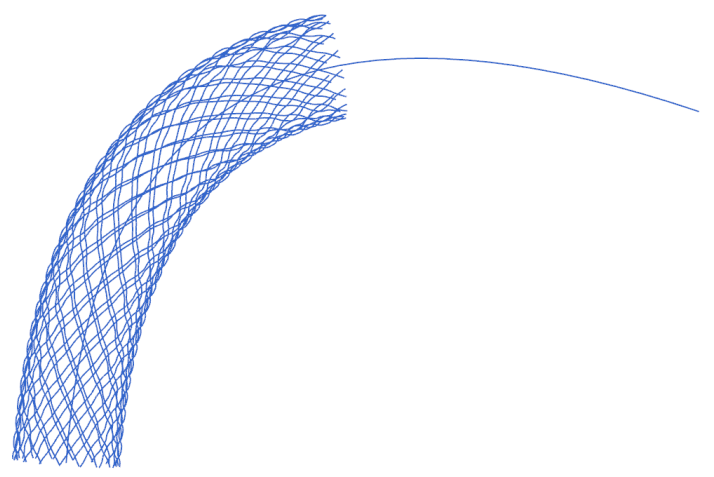

FiguRE 2. Undulation paths of the braided yarns modelled with a tapered cosine function following an arbitrary spline.

It should be noted that, due to the analytical modelling of the yarn undulation paths and cross sections, the numerical model assumes perfect in phase arrangement of the yarns. Hence there is total absence of any nesting or imperfections in the alignment of the yarns in the model.

\subsection{Material Model}

To take into account the meso-structure of the composite in the presented modelling approach it is distinguished between yarn behaviour (intra-yarn behaviour) and yarn-to-yarn interactions (inter-yarn behaviour).

The yarns (intra-yarn behaviour) are modelled utilising a transversely isotropic elastic material, taking into account the change of orientation along the yarn due to the yarn undulation. The material properties are determined from the constituents behaviour (matrix and fibres) using the MFH approach by Mori and Tanaka [5]. Furthermore, for modelling failure of the yarns (intra-yarn failure) Hashin's composite failure criteria [6] are implemented.

The determined yarn stiffness and strength properties can be found in Table 1.

Inter-yarn behaviour (yarn-to-yarn interactions) is being modelled using cohesive contact behaviour to prevent the yarns from separating during loading. By implementing a quadratic traction damage initiation

\begin{tabular}{llll}
\hline$E_{\|}(\mathrm{GPa})$ & 183 & $X^{T}(\mathrm{MPa})$ & 1810 \\
$E_{\perp}(\mathrm{GPa})$ & 20 & $X^{C}(\mathrm{MPa})$ & 669 \\
$\nu_{\| \perp}(-)$ & 0.21 & $Y^{T}(\mathrm{MPa})$ & 64 \\
$\nu_{\perp \perp}(-)$ & 0.46 & $Y^{C}(\mathrm{MPa})$ & 174 \\
$G_{\| \perp}(\mathrm{GPa})$ & 8.1 & $S^{L}(\mathrm{MPa})$ & 105 \\
$G_{\perp \perp}(\mathrm{GPa})$ & 6.9 & $S^{T}(\mathrm{MPa})$ & 105 \\
& & $\alpha(-)$ & 1 \\
\hline
\end{tabular}

TABLE 1. Yarn stiffness and strength properties.

criterion and energy based damage evolution, failure of the yarn-to-yarn interface is being accounted for. The used cohesive properties are specified in Table 2

\begin{tabular}{ll}
\hline Normal stiffness $(\mathrm{GPa})$ & 20 \\
Tangential stiffness $(\mathrm{GPa})$ & 6.9 \\
Damage initiation $(\mathrm{MPa})$ & 3 \\
Damage evolution $\left(\mathrm{mJ} / \mathrm{mm}^{2}\right)$ & $3 \cdot 10^{-3}$ \\
\hline
\end{tabular}

TABLE 2. Cohesive contact surface properties.

\section{EXPERIMENTAL}

Torsion tests were performed on thin walled single layer carbon fibre braided tubes using an INSTRON $8850(250 \mathrm{kN} / 2400 \mathrm{Nm})$ testing machine. The specimens had a total length of $300 \mathrm{~mm}$, an inner diameter of $70 \mathrm{~mm}$ and a wall thickness of $0.4 \mathrm{~mm}$. For gripping, glass fibre tabs with a thickness of $5 \mathrm{~mm}$ were glued to the specimens giving them an active length of $150 \mathrm{~mm}$. Fibre volume ratio (FVR) of the specimens was determined by burnt residue analysis to be approximately $53 \%$. An overview of the specimen geometry can be seen in Table 3 The torsion tests were conducted in rotation controlled mode with axial displacement restricted.

Full-field strain measurement was conducted using the DIC system Aramis 6.3 in 3D mode. To provide the necessary contrast for the Aramis measurement a speckle pattern was sprayed onto the specimens.

During testing initial failure was caused by torsional buckling of the structure (see Figure 3 followed by failure of the matrix. Upon further loading, buckling 


\begin{tabular}{ll}
\hline Braid FVR $(\%)$ & 53 \\
Yarn thickness $(\mathrm{mm})$ & 0.2 \\
Yarn width $(\mathrm{mm})$ & 4 \\
Yarn count (yarns/cm) & 2.15 \\
Layer thickness $(\mathrm{mm})$ & 0.4 \\
Braiding angle $\left(^{\circ}\right)$ & 45 \\
\hline
\end{tabular}

TABle 3. Yarn and braiding geometry of the test specimens.

of the structure developed but failure of the yarns was not observed.

A more detailed overview of the experimental results will be given in the following section.

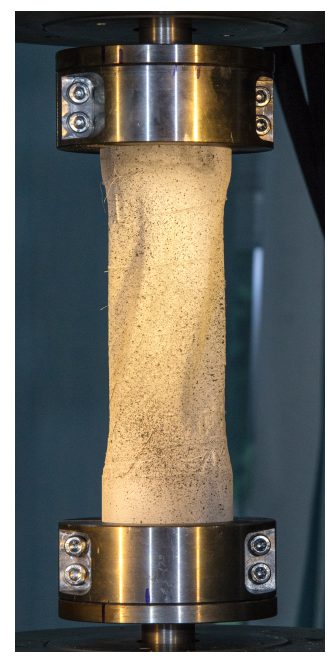

Figure 3. Torsion test of a tubular specimen with applied speckle pattern for full-field strain measurement and visible torsional buckling of the specimen.

\section{Results And Comparison}

The presented numerical model accurately predicts stiffness and strength of the braided tubular structure under torsional loading (a comparison is given in Table 4) and correlates well with the experimentally obtained data (see Figure 4).

\begin{tabular}{lll}
\hline & Experiment & Simulation \\
\hline $\begin{array}{l}\text { Torsional stiff- } \\
\text { ness }\left(\mathrm{Nm} /{ }^{\circ}\right)\end{array}$ & 137 & 140 \\
$\begin{array}{l}\text { Maximum } \\
\text { torque }(\mathrm{Nm})\end{array}$ & 48 & 48 \\
\hline
\end{tabular}

TABle 4. Comparison of experimental results and predicted values.

\subsection{Instability Behaviour}

In the numerical model, damage is initiated, during loading, at the yarn-to-yarn interface (inter-yarn level), leading to torsional buckling of the structure upon

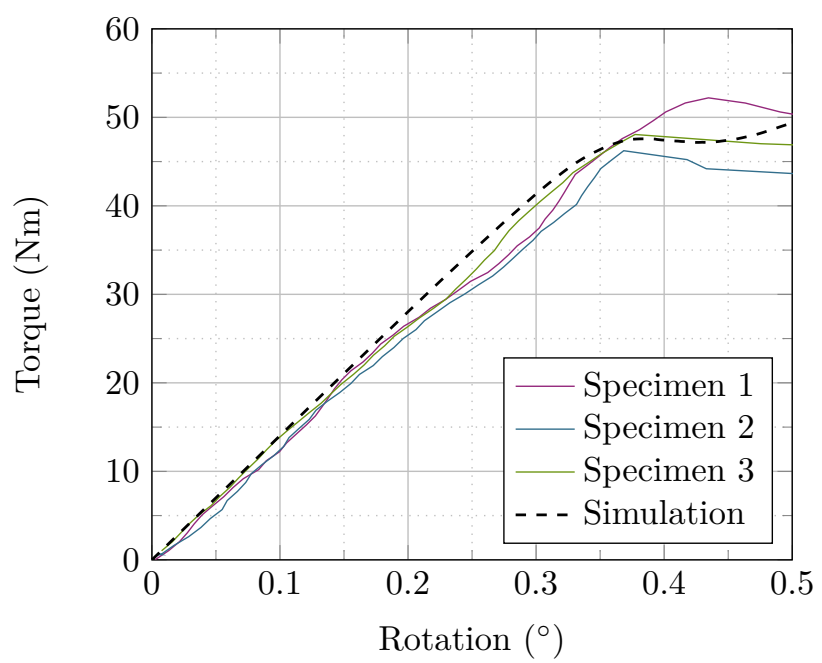

Figure 4. Comparison of the simulated and tested torque-rotation response.

further loading (see Figure 5(a). Failure at the intrayarn level does not develop in the simulated rotation range.

Similar instability and failure behaviour was observed during the experimental torsion tests (see Figure 5(b).

Comparing the displacement magnitude of the simulated structure and determined by DIC (Figure 5), the computed displacement generally follows the qualitative trend of the measured values while the maximum displacement of the buckles varies. Variations of the maximum displacement magnitude were also observed between the tested specimens though. As buckling under torsional load is generally prone to imperfections, this leads to the assumption that the differences originate mainly from imperfections in the specimens that were not taken into account in the numerical model.

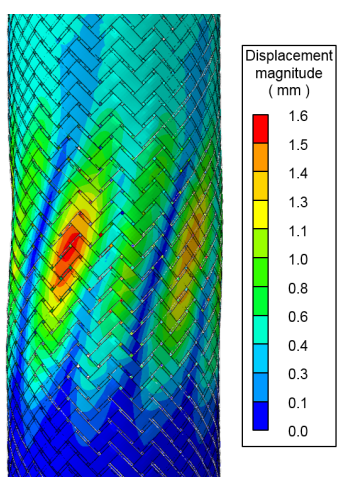

(a)

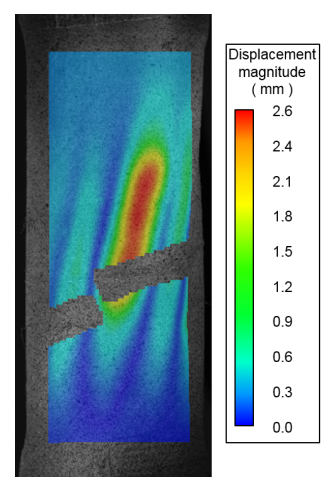

(b)
Figure 5. Buckling of the simulated structure (a) and of the tested specimen with an overlay of the DIC results (b), at a rotation angle of $0.65^{\circ}$. 


\subsection{Strain Distribution}

As the strains obtained by digital image correlation are measured on the surface of the specimen while the simulated strains are received at the yarn level, with the orientation following the undulation paths of the yarns, numerically and experimentally determined strains cannot be directly compared. Therefore a thin, low stiffness, and isotropic membrane layer was tied to the yarn surfaces at the outside of the tubular specimen in the numerical model. This does not influence the overall behaviour of the model but the strain distribution of the membrane layer now correlates to the surface strains of the specimen and hence it enables comparison with the digital image correlation results.

The strain in circumferential direction $\left(\varepsilon_{x}\right)$ on the surface of the sample examined for the numerical model and the DIC measurements (see Figure 6), shows a pattern of periodically repeating tensile and compressive strains as a consequence of the periodically crossing yarns. The measured strain generally follows the trend of the numerically predicted strain, with the simulation showing a more repetitive distribution.

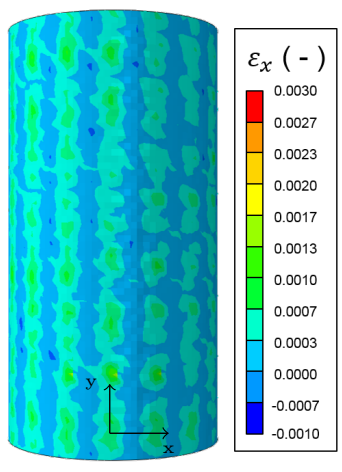

(a)

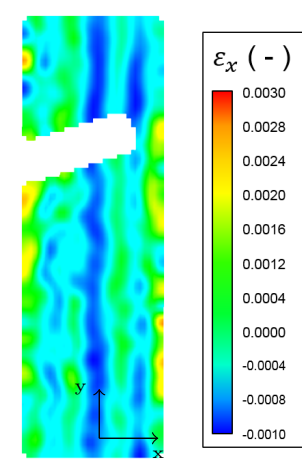

(b)
FiguRE 6. Strain distribution in the simulated structure (a) and experimentally obtained strain distribution (b) at a rotation angle of $0.25^{\circ}$, with $\varepsilon_{x}$ being the strain in circumferential direction of the cylinder.

To further compare the distribution, the measured and simulated strain profiles were traced along a circumferential line on the surface of the specimen, as can be seen in Figure 7

The period length of the computed strain oscillations is approximately $13.7 \mathrm{~mm}$, which corresponds to the circumferential distance between two recurring yarn crossings. Compared to the calculated strain profile, amplitude and period length of the measured strain profile show variations along the path.

It is again assumed that these variations arise from irregular spacing of the yarn crossings in the specimen, which causes variations of the local specimen wall thickness and the local stiffness. Such irregularities are not taken into account in the numerical model and hence cause the more repetitive strain profile of the numerical model.

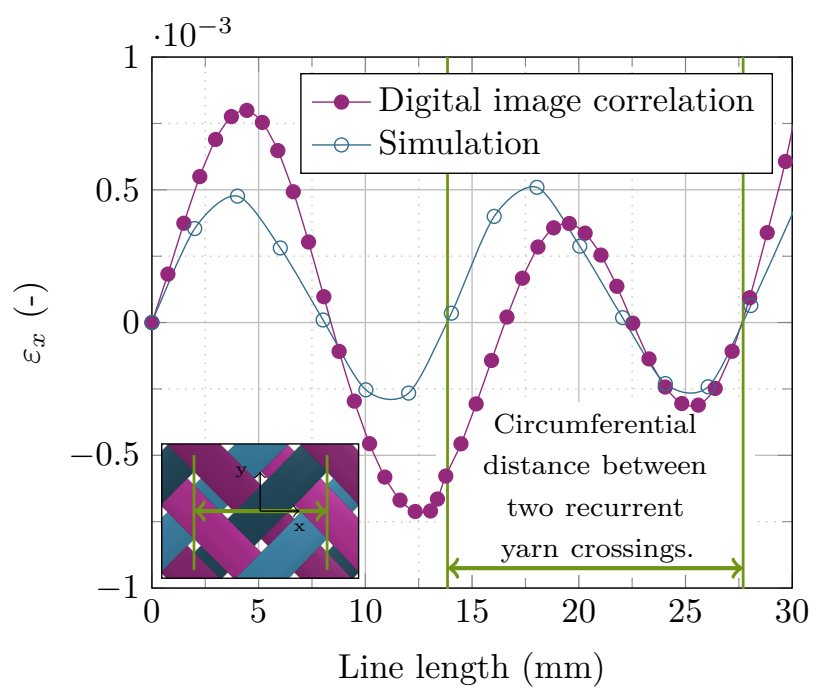

FIGURE 7. Comparison of the circumferential strain distribution $\left(\varepsilon_{x}\right)$ along a circumferential path (y-axis being parallel to the braiding direction).

\section{Conclusions}

The proposed modelling approach accurately reproduces the macroscopic response to loading and exhibits similar instability behaviour compared to experimental torsion tests and accurately predicts overall stiffness and strength of the component.

Further validation of the model was accomplished by utilising DIC, enabling full-field strain measurement on the surface of tubular braided samples.

Comparing the numerically and experimentally obtained local displacement and strain profiles, generally similar trends can be observed. Arising differences are assumed to be caused by imperfections in the tested samples that were not regarded in the numerical model. This states the necessity to further develop the numerical model to realistically represent imperfections in the structure and predict their influence on the performance of the component.

\section{LIST OF SYMBOLS}

$F V R$ fibre volume ratio [\%]

$E_{\|}$longitudinal elasticity modulus [GPa]

$E_{\perp}$ transversal elasticity modulus [GPa]

$G_{\| \perp}$ longitudinal shear modulus [GPa]

$G_{\perp \perp}$ transversal shear modulus [GPa]

$\nu_{\| \perp}$ longitudinal Poisson's ratio [-]

$\nu_{\perp \perp}$ transversal Poisson's ratio [-]

$X^{T}$ longitudinal tensile strength [MPa]

$X^{C}$ longitudinal compressive strength [MPa]

$Y^{T}$ transversal tensile strength [MPa]

$Y^{C}$ transversal compressive strength [MPa]

$S^{L} \quad$ longitudinal shear strength [MPa]

$S^{T}$ transversal shear strength [MPa] 
$\alpha$ contribution of shear stress to fibre failure [-]

$r$ current radius of the braiding layer $[\mathrm{mm}]$

$r_{\mathrm{o}}$ mean radius of the braiding layer $[\mathrm{mm}]$

$t \quad$ braiding layer thickness $[\mathrm{mm}]$

$\delta$ phase shift $[\mathrm{mm}]$

$\varepsilon_{x} \quad$ circumferential strain $[-]$

\section{ACKNOWLEDGEMENTS}

The current research is performed within the project number 5320343 / 849425 (ANDISIA) supported by the Austrian Research Promotion Agency, in cooperation with Teufelberger GmbH Austria and the University of Applied Sciences Upper Austria.

\section{REFERENCES}

[1] S. V. Lomov, D. S. Ivanov, I. Verpoest, et al. Full-field strain measurements for validation of meso-fe analysis of textile composites. Composites Part A: Applied Science and Manufacturing 39(8):1218 - 1231, 2008. Full-field Measurements in Composites Testing and Analysis, DOI:http://dx.doi.org/10.1016/j.compositesa.2007.09.011
[2] S. Neudorfer. Modelling and simulation of braided and filament-wound composite torsion-shaft, 2015. Master's thesis, Johannes Kepler Universität.

[3] M.-C. Miron, H. Katzinger, S. Neudorfer, et al. Mechanical testing and numerical predictions of the behavior of braided carbon fiber components. Procedia Structural Integrity 2:3593 - 3600, 2016. 21st European Conference on Fracture, ECF21, 20-24 June 2016, Catania, Italy, DOI:http://dx.doi.org/10.1016/j.prostr.2016.06.448

[4] E. Pitz, M.-C. Miron, Z. Major. Failure analyis of braided composite structures in torsional loading. Submitted for publication.

[5] T. Mori, K. Tanaka. Average stress in matrix and average elastic energy of materials with misfitting inclusions. Acta Metallurgica 21(5):571 - 574, 1973. DOI:http://dx.doi.org/10.1016/0001-6160(73)90064-3

[6] Z. Hashin. Failure criteria for unidirectional fiber composites. Journal of Applied Mechanics 47(2):329334, 1980. DOI:http://dx.doi.org/10.1115/1.3153664 